\title{
Triage and management of aortic emergencies during the coronavirus disease 2019 (COVID-19) pandemic: A consensus document supported by the American Association for Thoracic Surgery (AATS) and Asian Society for Cardiovascular and Thoracic Surgery (ASCVTS)
}

Christopher K. Mehta, MD, ${ }^{\text {a }}$ S. Chris Malaisrie, MD, ${ }^{a}$ Ashley N. Budd, MD, ${ }^{\mathrm{b}}$ Yutaka Okita, MD, PhD, Hitoshi Matsuda, MD, PhD, ${ }^{\mathrm{d}}$ Fernando Fleischman, MD, ${ }^{\mathrm{e}}$ Yuichi Ueda, MD, PhD, ${ }^{\mathrm{f}}$ Joseph E. Bavaria, MD, ${ }^{\mathrm{g}}$ and Marc R. Moon, MD

The coronavirus disease 2019 (COVID-19) pandemic has placed an unprecedented strain on hospitals worldwide, necessitating health care systems to triage patient care and redirect resources including personnel, equipment, and operating rooms. Aortic emergencies, including aortic dissection, rupture, and malperfusion syndromes, are resource-intensive and therefore can overwhelm a system already operating at maximal capacity. Although health care systems are necessarily shifting resources to address the COVID-19 pandemic, a contingency plan to continue to support aortic emergencies needs to remain in place. This document is meant to facilitate triage and management of these acute patients based on the best-available evidence.

1. How should hospitals manage COVID-19-positive or status unknown patients who present to their hospital with an aortic emergency?

From the a Division of Cardiac Surgery, Bluhm Cardiovascular Institute, and ${ }^{\mathrm{b}}$ Department of Anesthesiology, Northwestern University Feinberg School of Medicine, Chicago, Ill; ' Division of Cardiovascular Surgery, Kobe University Graduate School of Medicine, Kobe, Japan; ${ }^{\mathrm{d}}$ Department of Cardiovascular Surgery, National Cerebral and Cardiovascular Center, Osaka, Japan; ${ }^{\mathrm{e}}$ Department of Surgery, Keck School of Medicine, University of Southern California, Los Angeles, Calif; ${ }^{\mathrm{f}}$ Department of Cardiovascular Surgery, Nara Prefecture General Medical Center, Nara Prefectural Hospital Organization, Nara, Japan; ${ }^{\mathrm{g}}$ Division of Cardiovascular Surgery, Hospital of the University of Pennsylvania, Philadelphia, Pa; and ${ }^{\mathrm{h}}$ Division of Cardiothoracic Surgery, Washington University School of Medicine, St Louis, Mo.

Supported by the American Association for Thoracic Surgery and the Asian Society for Cardiovascular and Thoracic Surgery.

The contribution has been co-published in Journal of Thoracic and Cardiovascular Surgery [https://doi.org/10.1016/j.jtcvs.2020.06.004] and Asian Cardiovascular and Thoracic Annals [https://doi.org/10.1177/0218492320974505]. The contributions are identical except for minor stylistic and spelling differences in keeping with each journal's style.

Received for publication May 16, 2020; revisions received May 31, 2020; accepted for publication June 1, 2020; available ahead of print Oct 30, 2020.

Address for reprints: S. Chris Malaisrie, MD, Division of Cardiac Surgery, Bluhm Cardiovascular Institute, Northwestern University Feinberg School of Medicine, 676 N St Clair St, Arkes Pavilion, Suite 730, Chicago, IL 60611 (E-mail: Chris. Malaisrie@nm.org).

J Thorac Cardiovasc Surg 2021;161:48-53

0022-5223

Copyright (C) 2020 by The American Association for Thoracic Surgery and the The Asian Society for Cardiovascular and Thoracic Surgery. Published by Elsevier, Inc. This is an open access article under the CC BY license (http://creativecommons. org/licenses/by/4.0/).

https://doi.org/10.1016/j.jtcvs.2020.06.004

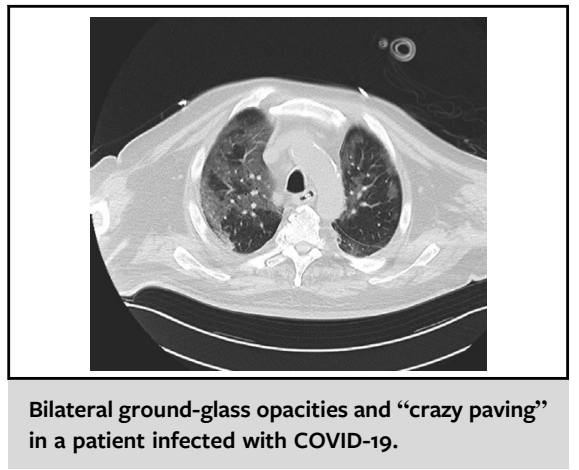

CENTRAL MESSAGE

An algorithmic approach to acute aortic emergencies during the COVID-19 pandemic can reduce the risk of exposure for patients and health care providers

See Commentaries on pages 54 and 55 .

a. Patients who present to an emergency department (ED) with an aortic emergency should initially be triaged into 1 of 3 categories based on their COVID-19 status by testing. A flowchart for triaging patients is demonstrated in Figure 1.

i. COVID-19-negative: Patients who have tested negative for COVID-19 should be managed by transfer to a routine surgical operating room (OR) and routine surgical intensive care unit (ICU) postoperatively.

1. Patients who test negative but have concerning signs/symptoms and/or radiologic findings concerning for infection on computed tomography scan (ie, possible false-negative; see Figure 2) 


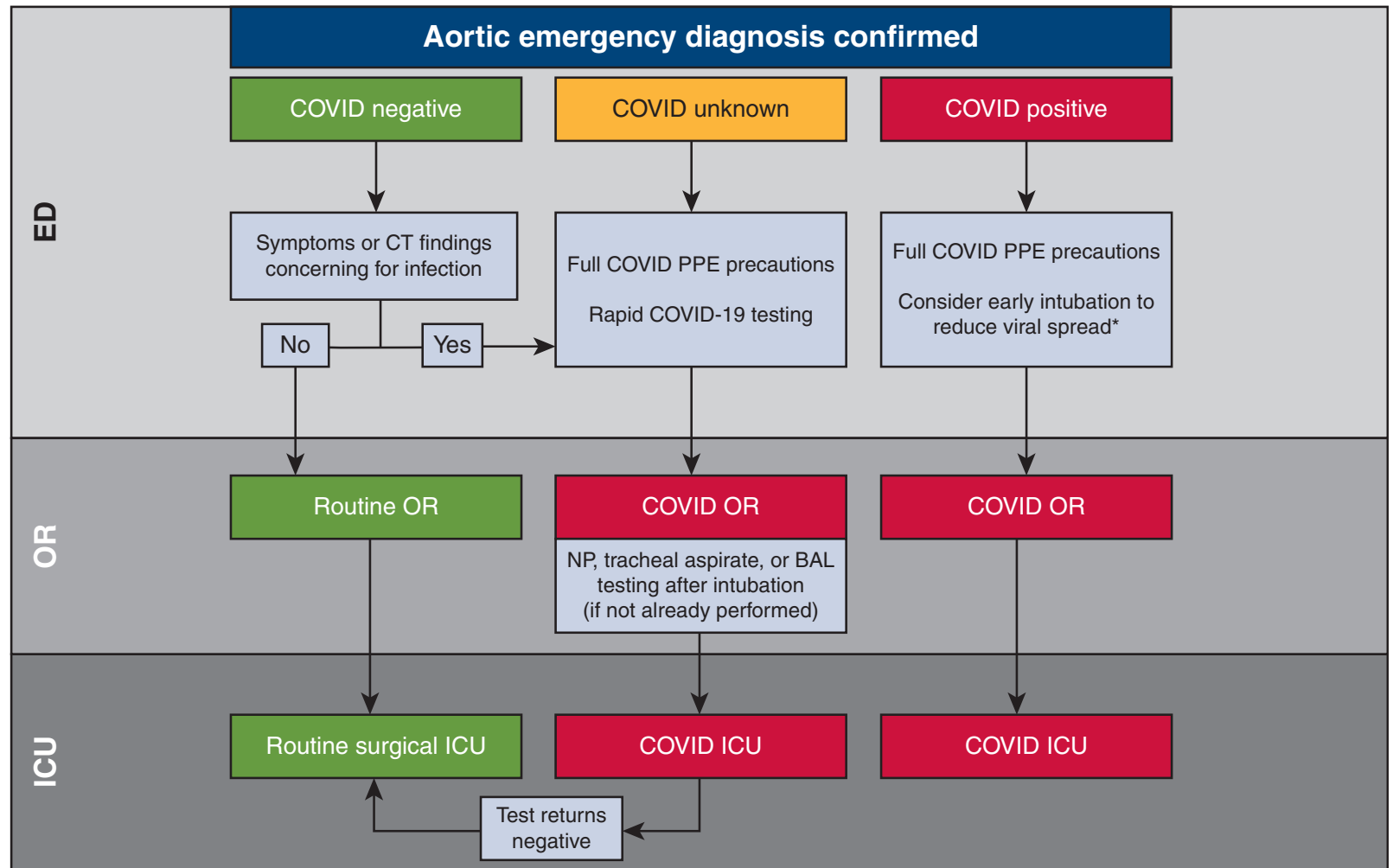

FIGURE 1. Algorithm for triaging patients from the emergency room based on COVID testing status. *Patients requiring high flow oxygen, non-invasive ventilation, or other oxygen source with high potential for aerosol-generation should be considered for early intubation in the ER in order to reduce viral spread during transport. $E D$, Emergency department; COVID-19, coronavirus disease 2019; $C T$, computed tomography; $P P E$, personal protective equipment; $O R$, operating room; $N P$, nasopharyngeal; $B A L$, bronchoalveolar lavage; $I C U$, intensive care unit.

should be treated as COVID-19 status unknown with full COVID-19 personal protective equip-

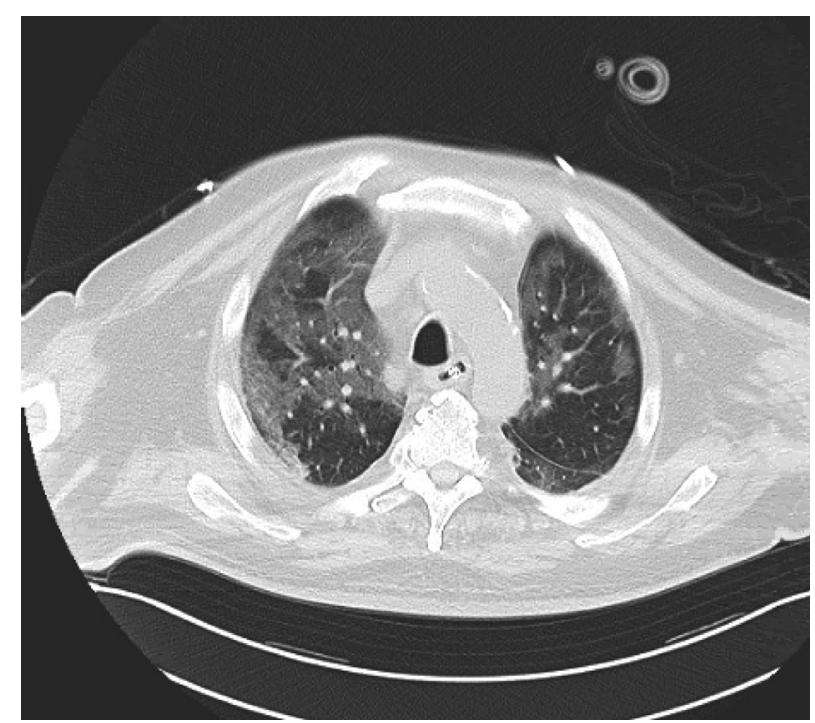

FIGURE 2. Computed tomography findings of the chest in a patient infected with COVID-19. Diffuse bilateral ground-glass opacities in a patient infected with COVID-19. Other characteristic findings include volume loss, atelectasis, and "crazy paving." ment (PPE) precautions and potentially retested using rapid testing.

ii. COVID-19-positive: Patients who have tested positive for COVID-19 should be managed with full PPE precautions and should be taken to a COVID-19-designated OR (capable of negativepressure airflow) and subsequently to a COVID19-designated ICU with close surgical team consultation.

1. Consider early intubation for symptomatic patients, ensuring appropriate PPE and in a negative pressure airflow room if able.

iii. COVID-19 status unknown: Patients for whom testing status is unknown or indeterminate should be handled with full PPE precautions and tested by rapid test in the ED.

1. Consider early intubation for symptomatic patients, ensuring appropriate PPE and in a negative-pressure airflow room if able.

2. If a sample needs to be obtained after intubation, consider tracheal aspirate or bronchoalveolar lavage, which have greater sensitivity than a nasopharyngeal swab, in accordance 


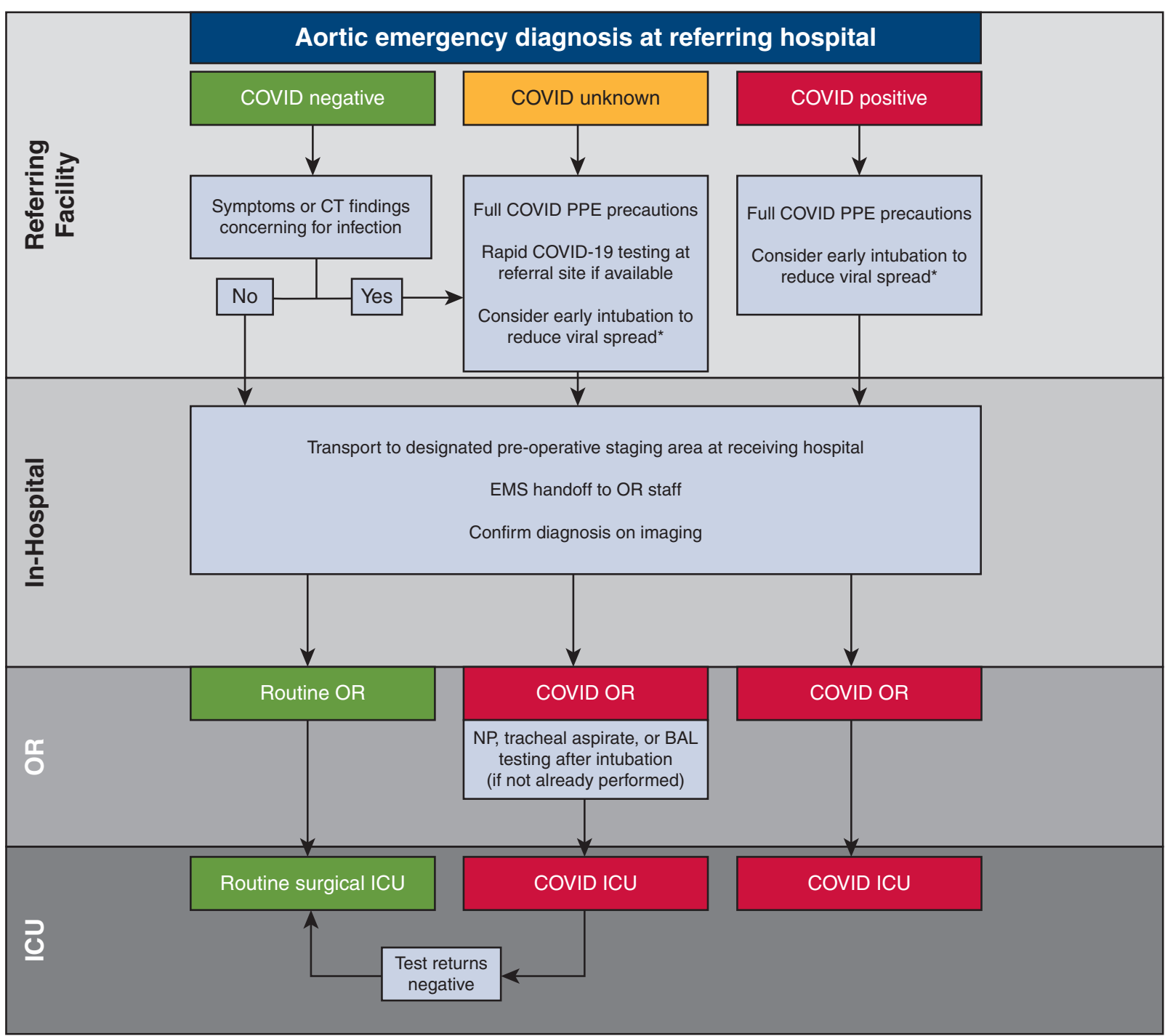

FIGURE 3. Algorithm for triaging patients from an external hospital. *Patients requiring high-flow oxygen, noninvasive ventilation, or other oxygen source with high potential for aerosol-generation should be considered for early intubation before transport to reduce viral spread during transport. COVID-19, Coronavirus disease 2019; $C T$, computed tomography; $P P E$, personal protective equipment; $E M S$, emergency medical services; $O R$, operating room; $N P$, nasopharyngeal; $B A L$, bronchoalveolar lavage; $I C U$, intensive care unit.

with the Society of Critical Care Medicine recommendations. ${ }^{1}$

3. Patients should be taken to a COVID-19designated ICU postoperatively until testing returns. If testing is negative, the patient should be transferred to a routine surgical ICU.

2. How should hospitals manage interfacility transfers of COVID-19-positive or status unknown patients?

a. The transfer of care to a greater level of care centers capable of handling aortic emergencies should continue during the COVID-19 pandemic but is necessarily dictated by local protocols. A flowchart for triaging patients from an external hospital is provided in Figure 3.

b. Patient transfer centers should alert the receiving treatment team of the patient's COVID-19 infection status. If the COVID-19 status is unknown, rapid testing, if available, should be performed by the transferring hospital before transport to the receiving facility.

c. Hospitals that have direct-to-OR transfer protocols for emergencies should continue to follow strict safety precautions to decrease the risk of transmission, and ensure the following:

i. Confirm transfer hospitals provide all pertinent medical records and imaging disks to decrease 
need for unnecessary repeat imaging testing at receiving facility.

ii. Limit family members from entering the receiving facility and establishing clear health care power of attorney contact information for decision-making.

d. Emergency medical services (EMS) and other transport personnel should wear appropriate recommended PPE (N95 or higher-level respirator or facemask when respirator unavailable, gloves, gown, eye protection) ${ }^{2}$ and take the following precautions:

i. Limit the number of personnel involved in the care and transfer of the patient.

ii. Minimize the spread of aerosol-generating equipment and procedures.

iii. Avoid transportation of the patient requiring highflow oxygen $(>15 \mathrm{~L} / \mathrm{min})$ or noninvasive ventilation, such as bi-level positive airway pressure or continuous positive airway pressure. Early intubation before transport should be considered for patients requiring-high flow oxygen $(>15 \mathrm{~L} /$ min) or noninvasive ventilation. ${ }^{3}$

iv. Patients may be transported on low-flow oxygen sources (such as nasal cannula, simple facemask, or non-rebreather mask). Face masks should be worn by the patient, including over any airway devices.

v. Have a clearly designated route to the patient transfer area; the receiving facility should have a representative available to escort the transport personnel to avoid unnecessary travel around the hospital.

3. What preoperative considerations should be taken to minimize risk to health care personnel for COVID-19positive or status unknown patients?

a. For surgical emergencies going directly to the OR, consider performing patient preoperative evaluation and any necessary procedures in the ED before transport to the OR (Table 1).

i. Provide bundled care treatment (blood draws, electrocardiogram evaluation, consent, and surgicalsite marking) to minimize personnel movement into and out of the room.

b. Hospitals that have direct-to-OR transfer protocols for emergencies should identify a preoperative surgical staging area where care can be transferred from EMS personnel to hospital personnel.

i. A surgical staging area may be a preoperative setting for centers with direct-to-OR abilities, or an ICU, depending on local protocols.

ii. EMS personnel should not transport patients directly to the OR, if possible, to avoid contamination. c. Appropriate PPE measures include the following:

i. Airway team: N95 masks or appropriate respirator, eye protection, gown, gloves, and head cover. A powered air-purifying respirator can also be considered when available.

ii. Nursing and surgical teams: N95 masks or appropriate respirator, eye protection, gown, gloves, and head cover.

d. Surgical procedures should ideally be performed in COVID-19-designated ORs that have the capability of providing negative-pressure airflow. Doffing of PPE should be performed adjacent to the OR doorway before leaving the room, or in a separate anteroom if available.

e. The minimum number of personnel required should be directly involved in patient care to reduce exposure. Team members at greater risk of COVID-19 infection should be identified and accommodated.

f. If the patient goes into cardiac arrest before procedure, standard advanced cardiovascular life support guidelines should be performed with the following modifications ${ }^{4}$ :

i. Chest compressions should be held during airway procedures to reduce aerosol generation and protect airway personnel.

ii. The minimal required personnel should be directly involved in patient care with a runner nearby to retrieve equipment and medications.

iii. In the event of suspected aortic catastrophe, early termination of resuscitative efforts should be considered.

g. Medical therapy and palliation are reasonable alternatives, particularly in patients with symptomatic COVID-19 infection. ${ }^{5}$

4. What are the important anesthetic considerations for COVID-19-positive and status unknown patients?

a. All precautions should be taken to minimize exposure to aerosol-generating procedures in the OR. Staff not involved in the management of the airway should wait outside the OR during this time.

b. In accordance with the World Health Organization and Centers for Disease Control and Prevention, aerosol-generating procedures should be performed in a negative-pressure airflow room.

c. Recommendations for endotracheal intubation include rapid sequence intubation, intubation performed by a highly experienced and efficient provider, and elective use of a video laryngoscope if available. ${ }^{1,6}$

i. Video laryngoscope allows the provider's face to be further away from the patient during 
intubation. ${ }^{7}$ In addition, a video laryngoscope may be beneficial in decreasing failed intubation attempts ${ }^{8}$ and increasing the success rate.

ii. The use of a high-efficiency hydrophobic filter should be interposed between the endotracheal tube and resuscitation bag or anesthesia circuit.

iii. Care should be taken to avoid contamination or cross-contamination after intubation.

d. If testing for COVID-19 was not yet achieved, a sample should be collected once in the OR.

i. The nasopharyngeal swab may be obtained; however, concerns have been expressed over the sensitivity of this sample. ${ }^{9}$ When the patient is intubated in the OR, a lower respiratory tract sample should be considered, in the form of a tracheal aspirate or bronchial alveolar lavage, in accordance with recommendations from the Society of Critical Care Medicine.

e. Ventilation of patients during the procedure should consider the pulmonary effects of patients infected with COVID-19.

i. Infected patients may present with acute respiratory distress syndrome (ARDS); however, some patients may have mild hypoxemia or be asymptomatic carriers.

1. Low tidal volume mechanical ventilation should be used to prevent further lung injury, as indicated in other types of ARDS. ${ }^{10,11}$

2. It is also reasonable to consider higher positive end-expiratory pressure as needed for hypoxemia per evidence-based ARDS protocols. ${ }^{10,12}$

f. The risk of performing an intraoperative transesophageal echocardiogram (TEE) in patients infected with COVID-19 is high.

i. Per the American Society of Echocardiography, the benefit of performing a TEE may outweigh the risk in a type A dissection in a COVID-19-positive or suspected patient. ${ }^{13}$ A case-by-case basis should be considered when determining whether to perform a TEE.

ii. Care should be taken to minimize contamination of surfaces while performing a TEE and appropriate PPE donned during the procedure.

5. What precautions should be taken by surgical personnel during the procedure?

a. Expeditious repairs requiring minimal operating and cardiopulmonary bypass times should be favored over complex operations. The priorities should be to safely address any life-threatening issues, to be efficient with resource use, and to minimize the risk of postoperative complications. $^{14}$ i. Endovascular options (if available and applicable to the clinical scenario) should be considered over open surgical procedures when possible.

b. Prepping and draping the patient should be done in usual sterile fashion, with emphasis on providing an appropriate drape barrier between anesthesia and surgical teams to minimize surgical personnel exposure to airway and TEE interventions.

c. Observing, nonessential personnel should not be present during the procedure to reduce the amount of personnel in close contact.

d. During the COVID-19 pandemic, there have been concerns over adequate blood supply for patients in some regions due to increased blood product use and blood donation shortages. Blood-conservation management should be used during surgical

TABLE 1. Suggested approach for the management of aortic emergencies during the COVID-19 pandemic

Preoperative considerations

Preoperative evaluation should be performed before transport to OR

A preoperative surgical staging area should be identified (for directto-OR protocols)

Appropriate PPE measures should be strictly followed for all personnel

Surgical procedures should ideally be performed in COVID-19designated ORs that have the capability of providing negative pressure airflow

The minimum number of personnel required should be directly involved in patient care

Anesthetic considerations

All precautions should be taken to minimize exposure to aerosolgenerating procedures

Recommendations for endotracheal intubation include rapid sequence intubation, an experienced provider performing the procedure, and elective use of a video laryngoscope

If testing for COVID-19 was not yet achieved, a sample should be collected once in the operating room

Ventilation of patients should follow ARDS protocols

Care should be taken during TEE examination, including appropriate PPE

Procedural considerations

Prepping and draping in usual sterile fashion with emphasis on an appropriate drape barrier between anesthesia and surgical teams

Expeditious repairs should be favored over complex repairs

Consider blood-conservation management strategies

Chest tubes should be checked for air leaks and secure connections

Postoperative care

Ideal postoperative care in a COVID-19-designated ICU

Extubation criteria should be carefully considered to mitigate risk of reintubation

$\overline{O R \text {, Operating room; COVID-19, coronavirus 2019; ARDS, acute respiratory distress }}$ syndrome; TEE, transesophageal echocardiogram; $P P E$, personal protective equipment; $I C U$, intensive care unit. 
procedures for aortic emergencies. The following measures should be considered where available:

i. intraoperative cell salvage ${ }^{15}$;

ii. modified ultrafiltration ${ }^{16}$;

iii. acute normovolemic hemodilution ${ }^{17}$;

iv. viscoelastography-guided transfusions ${ }^{18}$; and

v. ensuring good hemostasis to minimize blood product use and need to return to OR.

e. Chest tubes should be checked for air leaks and ensuring that all connections are secure to reduce exposure via contamination. ${ }^{19}$

6. What are the postoperative considerations for COVID19-positive and status-unknown patients?

a. Patients should ideally be transported to a COVID19-designated ICU with close consultation with the surgical team, unless hospital protocols dictate otherwise.

b. Timing and planning for extubation will be multifactorial, and attention should be given to the patient's hemodynamic stability, neurologic examination, respiratory status, and any postsurgical concerns.

i. Since extubation is also an aerosol-generating procedure, extubation should be performed in a negative-pressure airflow room with appropriate PPE and minimizing personnel in the room at time of extubation.

ii. Meticulous care should be given to ensure patients are adequately ready for extubation to minimize risk of reintubation.

c. Patients in whom COVID-status was initially unknown but later returns as negative should be moved to a routine surgical ICU setting.

\section{Conflict of Interest Statement}

Dr Malaisrie: Terumo, Cryolife, and Medtronic; Dr Fleischmnan: Terumo, Edwards Lifesciences, W. L. Gore, and Cook; Dr Bavaria: Terumo, W. L. Gore, Medtronic, and Cook Vascular; Dr Moon: Medtronic. All other authors reported no conflicts of interest.

The Journal policy requires editors and reviewers to disclose conflicts of interest and to decline handling or reviewing manuscripts for which they may have a conflict of interest. The editors and reviewers of this article have no conflicts of interest.

\section{References}

1. Alhazzani W, Moller MH, Arabi YM, Loeb M, Gong MN, Fan E, et al. Surviving sepsis campaign: guidelines on the management of critically ill adults with coronavirus disease 2019 (COVID-19). Crit Care Med. 2020;46:854-87.

2. Interim Guidance for Emergency Medical Services (EMS) Systems and 911 Public Safety Answering Points (PSAPs) for COVID-19 in the United States; 2020. Available at: https://www.cdc.gov/coronavirus/2019-ncov/hcp/guidance-forems.html. Accessed April 25, 2020.
3. Odor PM, Neun M, Bampoe S, Clark S, Heaton D, Hoogenboom EM, et al. Anaesthesia and COVID-19: infection control. Br J Anaesth. 2020;125:16-24.

4. Edelson DP, Sasson C, Chan PS, Atkins DL, Aziz K, Becker LB, et al. Interim guidance for basic and advanced life Support in adults, children, and neonates with suspected or confirmed COVID-19: from the emergency cardiovascular care committee and get with the guidelines( $(\mathrm{R})$ )-resuscitation adult and pediatric task forces of the American Heart Association in collaboration with the American Academy of Pediatrics, American Association for Respiratory Care, American College of Emergency Physicians, The Society of Critical Care Anesthesiologists, and American Society of Anesthesiologists: supporting organizations: American Association of Critical Care Nurses and National EMS Physicians. Circulation. 2020;141:e933-3.

5. Trimarchi S, Eagle KA, Nienaber CA, Rampoldi V, Jonker FHW, De Vincentiis C, et al. Role of age in acute type A aortic dissection outcome: report from the international registry of acute aortic dissection (IRAD). J Thorac Cardiovasc Surg. 2010;140:784-9.

6. Orser BA. Recommendations for endotracheal intubation of COVID-19 patients. Anesth Analg. 2020;130:1109-10.

7. Meng L, Qiu H, Wan L, Ai Y, Xue Z, Guo Q, et al. Intubation and ventilation amid the COVID-19 outbreak: Wuhan's experience. Anesthesiology. 2020;132: 1317-32.

8. Lewis SR, Butler AR, Parker J, Cook TM, Schofield-Robinson OJ, Smith AF. Videolaryngoscopy versus direct laryngoscopy for adult patients requiring tracheal intubation: a cochrane systematic review. Br J Anaesth. 2017;119: 369-83.

9. Xie C, Jiang L, Huang G, Pu H, Gong B, Lin H, et al. Comparison of different samples for 2019 novel coronavirus detection by nucleic acid amplification tests. Int J Infect Dis. 2020;93:264-7.

10. Brower RG, Lanken PN, MacIntyre N, Matthay MA, Morris A, Ancukiewicz M, et al. Higher versus lower positive end-expiratory pressures in patients with the acute respiratory distress syndrome. N Engl J Med. 2004;351:327-36.

11. Fan E, Del Sorbo L, Goligher EC, Hodgson CL, Munshi L, Walkey AJ, et al. An official American Thoracic Society/European Society of Intensive Care Medicine/Society of Critical Care Medicine clinical practice guideline: mechanical ventilation in adult patients with acute respiratory distress syndrome. Am J Respir Crit Care Med. 2017;195:1253-63.

12. Mercat A, Richard JC, Vielle B, Jaber S, Osman D, Diehl J-L, et al. Positive end-expiratory pressure setting in adults with acute lung injury and acute respiratory distress syndrome: a randomized controlled trial. JAMA. 2008;299: 646-55.

13. Nicoara AM, Maldonado Y, Kort S, Swaminathan M, Mackensen GB. Specific considerations for the protection of patients and echocardiography service providers when performing perioperative or periprocedural transesophageal echocardiography during the 2019 novel coronavirus outbreak: council on perioperative echocardiography supplement to the statement of the American Society of Echocardiography. J Am Soc Echocardiogr. 2020;33:666-9.

14. Waterford SD, Gardner RL, Moon MR. Extent of aortic replacement in Type A dissection: current answers for an endless debate. Ann Thorac Surg. 2018;106: 1246-50.

15. Carless PA, Henry DA, Moxey AJ, O’Connell D, Brown T, Fergusson DA. Cell salvage for minimising perioperative allogeneic blood transfusion. Cochrane Database Syst Rev. 2010:CD001888.

16. Torina AG, Silveira-Filho LM, Vilarinho KA, Eghtesady P, Oliveira PPM Sposito AC, et al. Use of modified ultrafiltration in adults undergoing coronary artery bypass grafting is associated with inflammatory modulation and less postoperative blood loss: a randomized and controlled study. J Thorac Cardiovasc Surg. 2012;144:663-70.

17. Barile L, Fominskiy E, Di Tomasso N, Alpizar Castro LE, Landoni G, De Luca M, et al. Acute normovolemic hemodilution reduces allogeneic red blood cell transfusion in cardiac surgery: a systematic review and meta-analysis of randomized trials. Anesth Analg. 2017; 124:743-52.

18. Kuiper G, van Egmond LT, Henskens YMC, Roekaerts PM, Maessen JG, Ten Cate $\mathrm{H}$, et al. Shifts of transfusion demand in cardiac surgery after implementation of rotational thromboelastometry-guided transfusion protocols: analysis of the HEROES-CS (HEmostasis Registry of patiEntS in Cardiac Surgery) observational, prospective open cohort database. J Cardiothorac Vasc Anesth. 2019;33: 307-17.

19. Bilkhu R, Viviano A, Saftic I, Billè A. COVID-19: Chest Drains With Air LeakThe Silent 'Super Spreader'?; 2020. Available at: https://doi.org/10.25373/ ctsnet.12089130.v1. Accessed May 14, 2020. 